\title{
What causes the variations of the peak intensity of CIR accelerated energetic ion fluxes?
}

\author{
E. Keppler \\ Max-Planck-Institut für Aeronomie, D-37191 Katlenburg-Lindau, Germany \\ Received: 23 May 1997 / Revised: 11 December 1997 / Accepted: 12 May 1998
}

\begin{abstract}
The variation of the peak intensity of energetic ions accelerated at CIR related shocks in the interplanetary medium as observed by instruments on board of ULYSSES during its pass towards the south polar region and from the north polar region back to its aphelium is discussed. From ULYSSES measurements alone it cannot be decided whether the observed variation is a function of latitude or of radial distance, as its orbit changes distance and latitude at the same time. Therefore ULYSSES data is compared with earlier observations by the PIONEER and VOYAGER spacecraft and concluded that the major part of the observed variation of the peak intensity seems to be due to a radial distance change, on to which, however, at higher latitudes a latitude dependent feature is superimposed.
\end{abstract}

Key words. Interplanetary physics (Energetic particles; interplanetary shocks; general)

\section{Introduction}

It is well known that corotating structures are formed by fast solar wind streams ramming into slow solar wind ahead. When the fast flow meets the slow wind ahead, a complicated interaction region is formed, called, as the structure corotates with the Sun, corotating interaction region (CIR). A forward shock (FS, which from the existing geometry propagates towards lower latitudes) is driven into the upstream slow wind region, and a reverse shock (RS; which moves to higher latitudes) into the fast upstream region. Energetic ions are accelerated at both shocks, the FS and the RS, electrons to energies above $100 \mathrm{keV}$ apparently only at the RS. Also, the RS appears to be the more efficient particle accelerator. This feature is presently not understood. Beyond about $-30^{\circ}$ heliolatitude, i.e. after the $\mathrm{S} / \mathrm{C}$ had left the streamer belt, the
FS could no longer reach the $\mathrm{S} / \mathrm{C}$, also particles accelerated there were no longer observed. The RSs, however, were observed in a regular manner until about $-41^{\circ}$ (Keppler et al., 1995). A few shocks have been observed when ULYSSES had moved beyond $-41^{\circ}$, but it is not clear whether these shocks were related to CIRs at all (Phillips et al., 1995). Despite the fact that shocks did not regularly propagate to much higher latitudes than $-41^{\circ}$, energetic particle were observed up to as high as $-68^{\circ}$, obviously accelerated at the RS, because they still showed signatures as observed at RS only.

As was shown by Fraenz et al., (1994), the three dimensional anisotropy of CIR accelerated particles is field aligned and directed towards the Sun. So the particles apparently move along the magnetic field lines and may, thus, also arrive in higher heliographic latitudes, once magnetic flux tubes connect the spacecraft to the acceleration region. This seems to agree with a topology suggested by Fisk (1996) who inferred a non radial expansion for field lines originating in higher heliographic latitudes to much lower latitudes due to the differential rotation of the high latitudes solar photosphere, where these field lines are presumably anchored in the supergranulation. In the picture described by Fisk (1996) the observed particles would have moved along a magnetic flux tube which temporarily connected the observer to the shock surface. So these observed CIR related fluxes encountered at high latitudes are due to the topological structure of the heliospheric magnetic fields, and thus, latitude dependent. This model has been used by Roelof and Simnett (1996) to explain the increasing time lag between shock accelerated energetic electrons $(\mathrm{E}>50 \mathrm{keV})$ and ions once the shocks no longer passed the $\mathrm{S} / \mathrm{C}$. Absence of such particles would mean that the flux tube no longer connected to the shock.

The PIONEER 10/11 observations have indicated that the intensity of accelerated energetic particles increases with distance from the Sun until 4-5 AU, but decreases afterwards. Van Hollebeke et al., (1978) 
derived a radial gradient of $350 \% \pm 150 \% / \mathrm{AU}$ between 0.3 and $1 \mathrm{AU}$, and a somewhat smaller gradient between 1 and 4 AU (20...200\%/AU). Beyond 4 AU they find the gradient to become negative between 4 and 10 AU $(-40 \ldots-100 \% / A U)$. They also looked into the possibility of isolating a latitudinal effect but could only conclude that if it existed it would be much smaller than the radial gradient. The present study is intended to compare ULYSSES findings with those of the earlier ones.

\section{Instrumentation}

This report is based on observations obtained with the EPAC Instrument on ULYSSES. The EPAC instrument consists of four identical three-element semiconductor telescopes mounted at different angles relative to the spacecraft spin axis, so that, by virtue of the spin rotation about $80 \%$ of the sphere is sampled in 32 bins. By means of the $(\mathrm{dE} / \mathrm{dx})$-E-technique elements up to iron can be separated. The energy ranges covered are 0.3 to $1.5 \mathrm{MeV}$ protons, and 0.4 to $6 \mathrm{MeV} / \mathrm{N}$ for heavier ions. Electrons are measured in two channels $(0.1<\mathrm{E}<0.38 \mathrm{MeV}$, "ELL", and $\mathrm{E}>0.18 \mathrm{MeV}$, "ELH"), spin averaged for each telescope. A detailed description of the instrument can be found in Keppler et al. (1992).

\section{Observations}

In Fig. 1 there is a summary of helium ion flux observations after ULYSSES left the Jovian environment. Since then it has moved along its highly inclined interplanetary orbit back to its aphelium. The fast latitude scan from pole to pole in the perihelion phase has been omitted for clarity. Thus Fig. 1 shows the whole latitudinal/radial scans from the ecliptic plane in 5.3 AU to the south polar regions at 2.3 AU, and from the north polar regions back into the ecliptic plane. The intensities of all ion species (helium ions, energy range 0.4-1.6 MeV/N, are shown; protons and oxygen, not shown here, in similar energy ranges behave similarly) rise and fall in a rhythm of about 26.3 days, the apparent solar rotation period. A further restriction to the region where shocks passed the $\mathrm{S} / \mathrm{C}$ was made, so that the particle fluxes under discussion were accelerated at nearby shocks and propagation effects must not be taken into account, i.e. to the streamer belt region. The variations in the Southern Hemisphere $(\mathrm{SH})$ were obviously related to the persistent existence of a coronal hole which extended to quite low latitudes.

In the Northern Hemisphere (NH) CIR accelerated energetic ions were picked up again on day 1/1996 near $+52^{\circ}, 3.1 \mathrm{AU}$ from the Sun. Apparently this occurred when, during Carrington rotation 1901, again an extension of the north polar coronal hole down to $20^{\circ}$ latitudes appeared. Following rotation 1906 another extension of the polar coronal hole appeared at a different solar longitude; correspondingly the CIR related particle fluxes appeared at a different phase, i.e. some 10 days later, compared to the previous repetition cycle. From this time onwards, the intensities of the CIR-events became continuously larger as the S/C moved further out and towards lower latitudes. Only after day 250/1996 have shocks been observed again to pass the $\mathrm{S} / \mathrm{C}$ (R. J. Forsyth, private communication). In fact, as shown in Fig. 2, and for clarity also in Fig. 4, if the data (received below $40^{\circ}$ latitude), shown in Fig. 1, are plotted versus radial distance, the variation of the peak intensities of the two passes seem to match quite well.

For comparison the same data as shown in Fig. 2 is shown in Fig. 3 as a function of heliographic latitude. It can be seen that a preference for either one is not possible from the ULYSSES data alone. It is apparent that in the NH the peak intensity of the accelerated particles was about an order of magnitude lower compared to the southern pass. Also, there have been

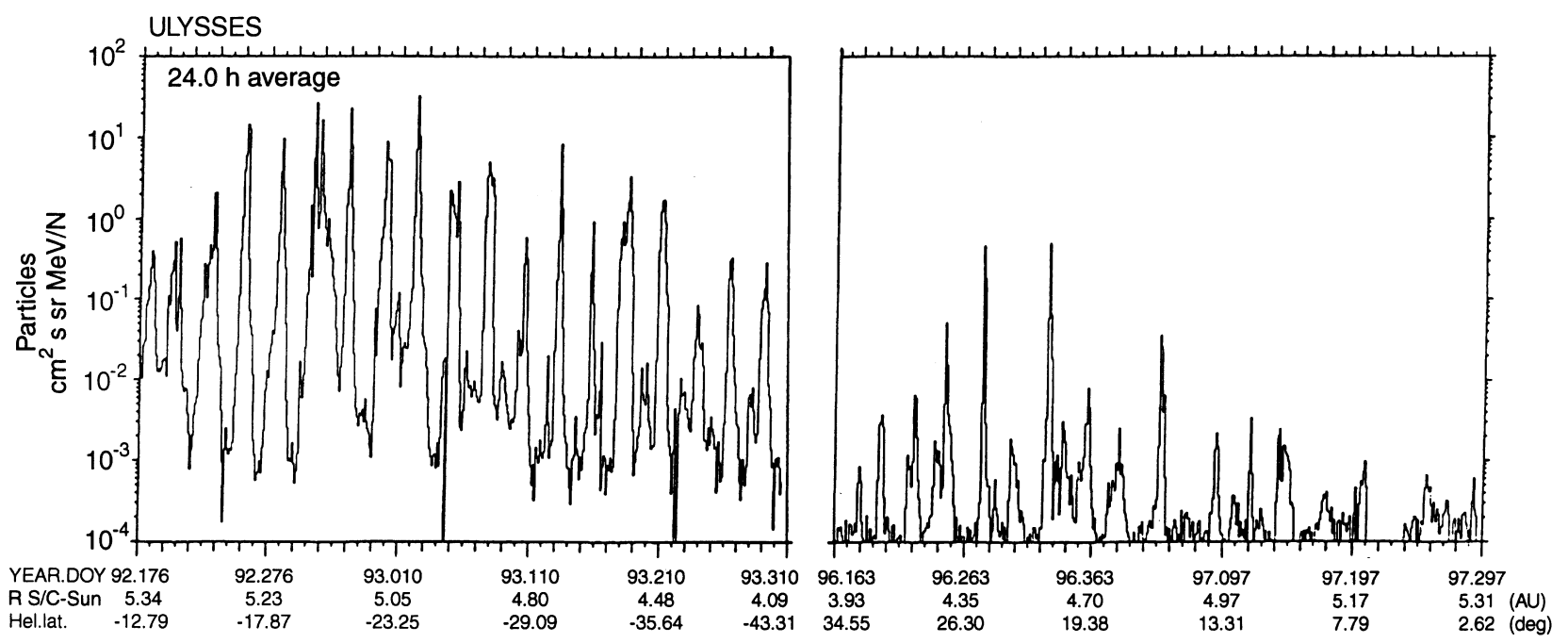

Fig. 1. Helium ion fluxes $(0.4 \mathrm{E} / \mathrm{N} 1.6 \mathrm{MeV} / \mathrm{N})$ versus time as measured by the ULYSSES-EPAC instrument 


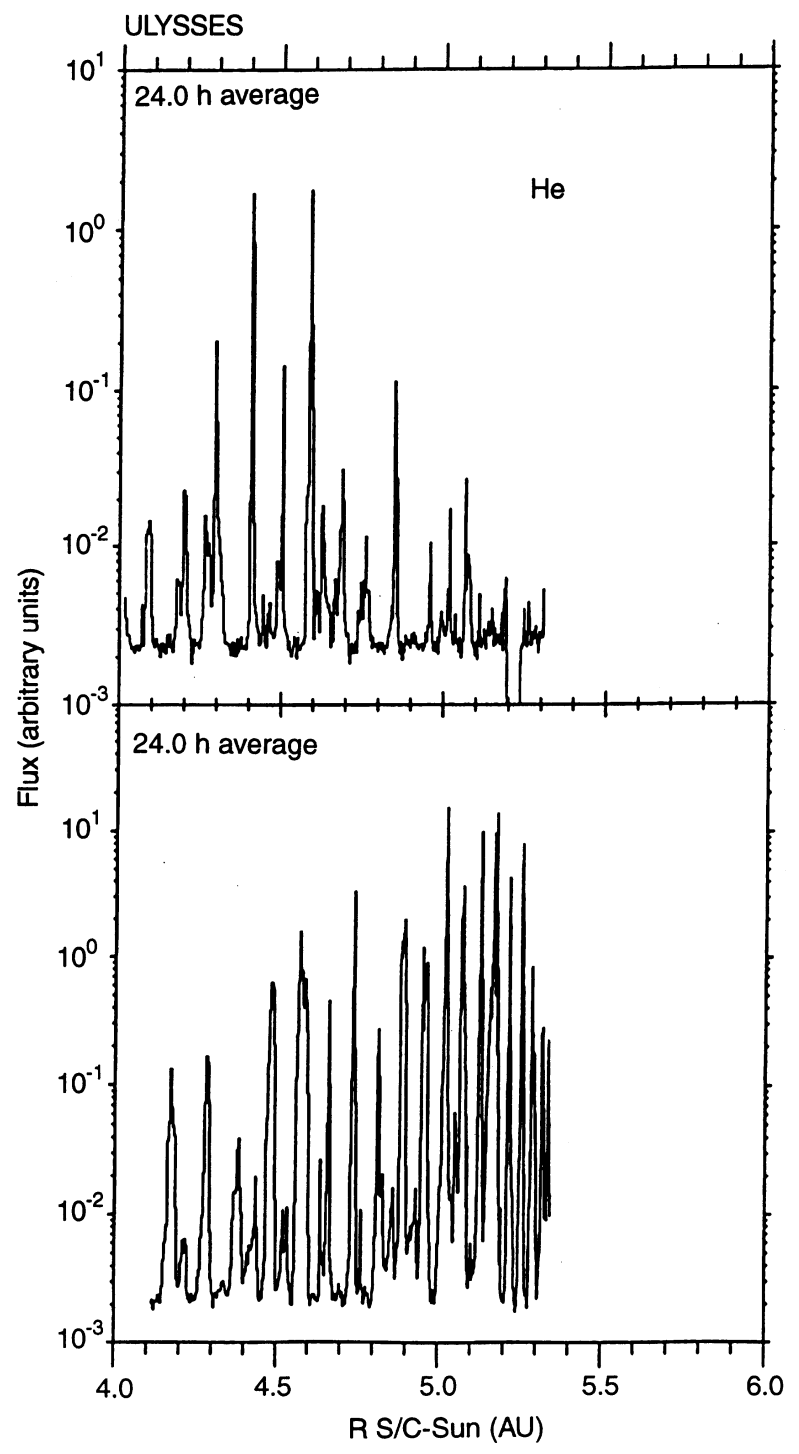

Fig. 2. Same data as shown in Fig. 1, however, plotted against radial distance from the Sun (upper panel, inbound pass; lower panel, outbound pass)

periods, where CIRs were not observed, consistent with the changes in the topography of the polar coronal holes on the solar disk. It is therefore not the number of CIRs and associated energetic particle flux increases observed in both hemispheres which are to be compared. The peak intensity of an event has been taken as the criterion rather than the integral over the observed flux because the latter might have been recorded during varying propagation/connection conditions. The peak flux appears within a short time only and should, thus, reflect optimum connection to the acceleration region, although the parameters which influence the peak intensity of the particle fluxes are at present not well understood. It has been argued that it is dependent on the Alfvén Mach number and on the angle between the shock normal and the upstream magnetic field (Classen et al., 1997). Other views refer to solar activity being responsible for providing enough seed particles (Desai et al., 1997).
From the observed data, however, we are led to consider the gradient of change of the peak intensities, either with latitude or with radial distance, to be the salient feature. For the southern pass for the flux increases $-40 \%$ per degree is found and for the northern pass $30 \%$ per degree. If interpreted as a function of radial distance from the Sun the numbers for both passes are 500-600\%/AU and $>1000 \% / \mathrm{AU}$, respectively.

On the other hand ULYSSES observed a decay of the peak intensities on both passes beyond 4.6 AU $(\mathrm{NH}$; $-100 \% / \mathrm{AU})$ and beyond $5 \mathrm{AU}(\mathrm{SH},>-1000 \% / \mathrm{AU})$. During this period latitude did not change too much, so that the Ulysses results compare directly with Pioneer and Voyager results. A similar feature ( -40 to $-100 \%$ / AU) has been reported by van Hollebeke et al. (1978) again beyond $5 \mathrm{AU}$. Their measurements extended to about 10 AU, while ULYSSES measurements extend only to about 5.3 AU. All numbers quoted here are, however, crude figures only.

\section{Discussion}

Inspection of Fig. 1 shows that CIR related particles and the accelerating shocks are observed to heliographic latitudes of about $-41^{\circ}$ with decreasing intensity. The PIONEER 10/11 observations were obtained in the ecliptic plane (van Hollebeke et al., 1978), and have been interpreted as showing a radial gradient. If this inference is true, then also the ULYSSES observations could be interpreted as showing a radial gradient.

Further, Gold et al. $(1985,1988)$ reported data from the VOYAGER spacecraft obtained at an even larger distance from the Sun to show decreasing peak intensities when the $\mathrm{S} / \mathrm{C}$ moved away from the ecliptic plane. This was observed during a quiet period (with respect to solar activity) which lasted for about 200 days so that the CIR observations were not masked by other effects.

In view of the order of magnitude difference in the peak intensities between the $\mathrm{SH}$ and the $\mathrm{NH}$ passes we note that this cannot be easily explained. The formation of CIRs is a consequence of the interaction of fast and slow solar wind; the speed of the fast wind is independent of the solar activity cycle, the speed of the slow wind might vary with the low latitude activity, but typically in a range such as $300-450 \mathrm{~km} / \mathrm{s}$. Therefore, also the existence of CIRs cannot depend on solar activity but only on the structure of the coronal holes from which fast wind originates. A boundary of the polar coronal hole along constant solar latitude (and with no equatorial holes present) would not produce a fast wind behind the slow wind and thus CIRs would not be formed at all. One reason for the difference between $\mathrm{SH}$ and $\mathrm{NH}$ energetic particle observations might be caused by the very different structure of the coronal holes during the two periods. The observation of CIR related effects in the interplanetary medium during high solar activity might, however, be masked by other effects such as CMEs or flares. 

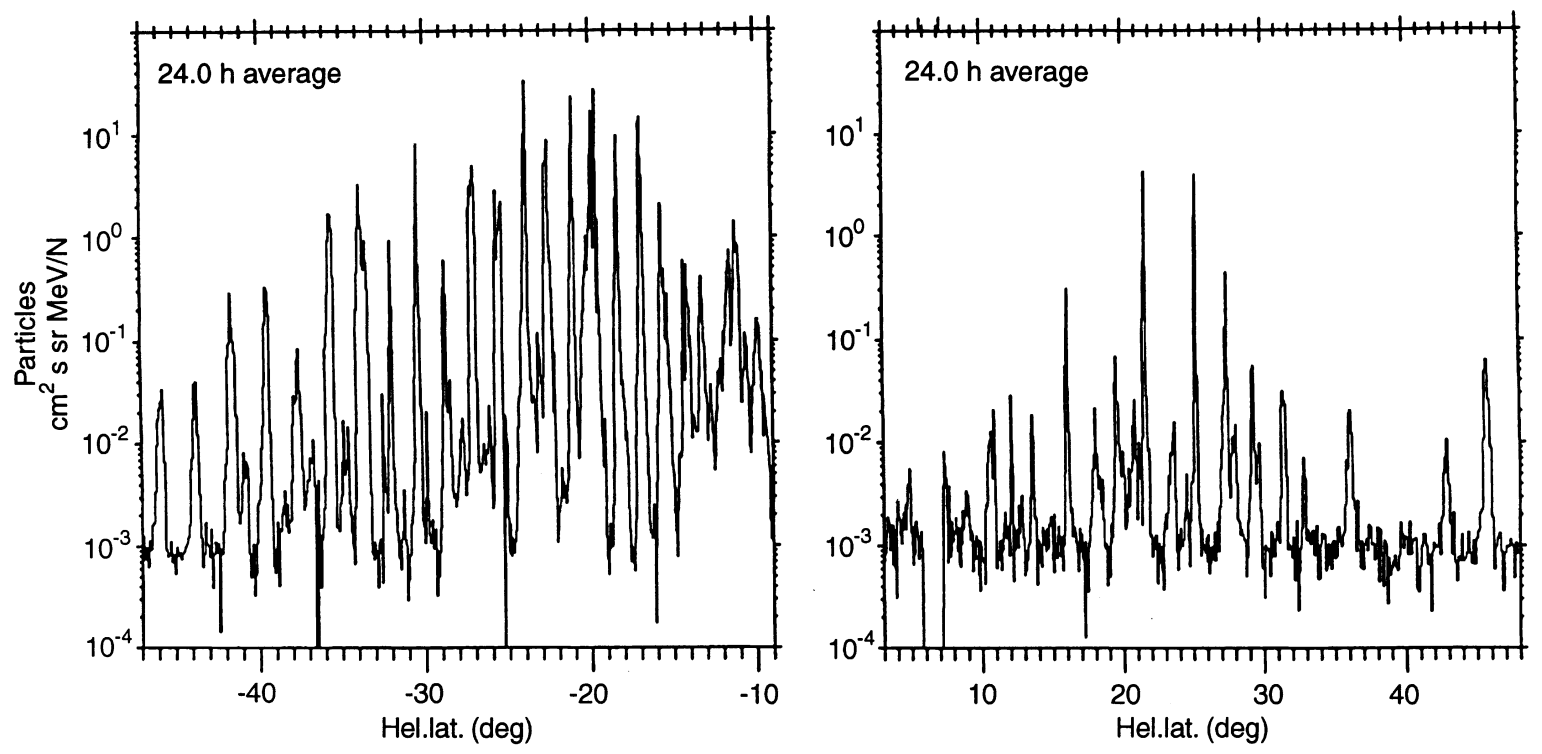

Fig. 3. Same data, plotted versus heliomagnetic latitude (left panel, inbound; right panel, outbound)

As CIRs move to greater distances, material continues to flow through the shocks so that the compression ratio between the shocks and their interface increases quite naturally as the shocks reach larger distances. For travelling shocks Lopate (1989) has shown that efficient particle acceleration requires three conditions to be fulfilled: presence of precursor waves, compression ratios $>3$, and scattering centres converging with some minimum speed. This might be a hint to understand why the peak intensities reach a maximum in 4 to $5 \mathrm{AU}$ distance from the Sun.

A further hint, as shown by Keppler et al. (1996) is that the spectral slope of the energy spectra of the accelerated ions remains the same during all CIR events. Also the $\mathrm{p} / \mathrm{He}$ ratio at $\mathrm{RS}$ is $<10$ throughout the observed RS events. As suggested by Simnett et al. (1995), this might be due to accelerated He pick up ions. The density of these pickup ions (which were interstellar neutrals before) in the Heliosphere is, however, a function of distance from the Sun, not latitude dependent. So this is another argument in favour of our plea for distance controlled effects.

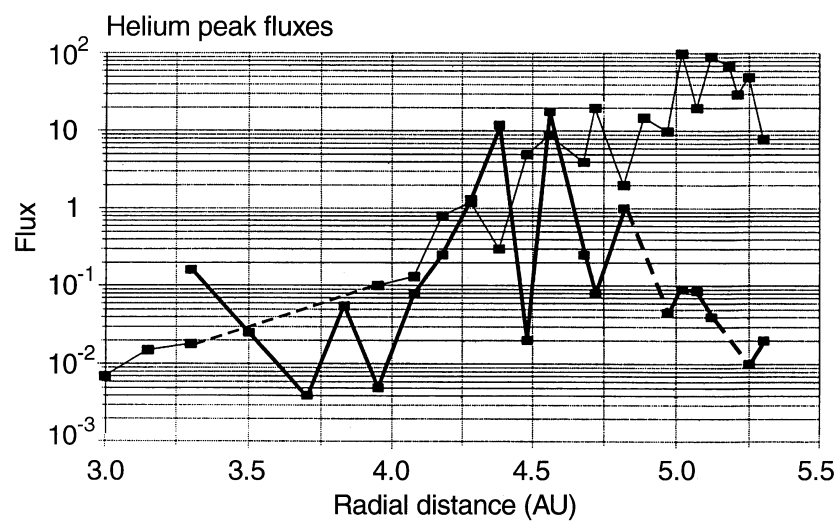

Fig. 4. Peak fluxes of energetic helium ions plotted versus radial distance from the Sun. Thin lines, inbound; thick lines, outbound
If we take all facts together, we are forced to assume that the variation of the peak intensity of CIR accelerated energetic particles is basically a function of radial distance. That part of the observed flux increases which occurred during times when the shocks no longer reached the $\mathrm{S} / \mathrm{C}$ is obviously latitude dependent. As mentioned in the introduction this part is interpreted in terms of the topology of the heliospheric magnetic fields. The intensity might then be further reduced due to the longer path travelled by these particles, which causes adiabatic deceleration and losses due to cross field diffusion. It is this part of the ULYSSES observations which could then be interpreted as showing a latitudinal dependence in the way as has been done by Simnett and Roelof (1995) and Roelof et al. (1996).

Acknowledgements. I. Schrader helped me in preparing the final version of the manuscript. This work was supported in part by DARA (grant 50 ON 91050) and by the Max-Planck-Gesellschaft zur Förderung der Wissenchaften e.V.

Topical Editor R. Schwenn thanks E. C. Roelof and another referee for their help in evaluating this paper.

\section{References}

Classen H. T., G. Mann, E. Keppler, Particle acceleration efficiency and MHD characteristics of CIR related shocks, Astron. Astrophys., accepted for publication 1997.

Desai M. I., R. G. Marsden, T. R. Sanderson, A. Balogh, R. J. Forsyth, J. T. Gosling, Energetic particle acceleration at corotating interaction regions; ULYSSES results, Geophys. Res. Lett., 24, 1155-1158, 1997.

Gold R. E., L. J. Lanzerotti, C. G. Maclennan, and A. M. Krimigis, Latitude dependence of corotating shock acceleration, Proc. 19. Int. Conf. Cosmic Rays, 4, 186-189, 1985.

Gold R. E., R. B. Decker, S. M. Krimigis, L. J. Lanzerotti, and C. G. Maclennan, The latitude and radial dependence of shock acceleration in the heliosphere, J. Geophys. Res., 93, 991-996, 1988.

Fisk L. A., Motion of the foot points of heliospheric magnetic field lines at the Sun: implications for recurrent energetic particle 
events at high heliographic latitudes, J. Geophys. Res., 101, 15547-15553, 1996.

Fraenz M., E. Keppler, N. Krupp, M. K. Reuss, and J. B. Blake, The elemental composition in energetic particle events at high heliospheric latitudes, Space Sci. Rev., 72, 339-342, 1995.

Keppler E., J. B. Blake, D. Hovestadt, A. Korth, J. J. Quenby, G. Umlautt, and J. Woch, The ULYSSES: energetic particle composition experiment EPAC, Astron. Astroph. Suppl. Ser., 92, 317-331, 1992.

Keppler E., M. Fraenz, A. Korth, M. K. Reuss, J. J. Quenby, J. B. Blake, M. Witte, Observations of energetic particles with EPAC on ULYSSES in polar latitudes of the heliosphere, Science, $\mathbf{2 6 8}$, 1013-1016, 1995.

Keppler E., B. Drolias, M. Fraenz, A. Korth, M. K. Reuss, J. B. Blake, and J. J. Quenby, The high latitude pass of ULYSSES: energetic particle observations with EPAC, Astron. Astroph., 316, 464-480, 1996.

Lopate C. J., Electron acceleration of relativistic energies by travelling interplanetary shocks, Geophys. Rev., 94, 9995$10010,1989$.
Phillips J. L., S. J. Bame, A. Barnes, N. L. Barraclaugh, W. C. Feldmann, B. E. Goldstein, J. T. Gosling, G. W. Hoogeveen, D. J. McCornas, M. Neugebauer, and S. T. Suess, ULYSSES solar wind plasma observation from pole to pole, Geophys. Res. Lett., 22, 3301-3304, 1995.

Roelof E. C., G. M. Simnett, and S. J. Tappin, The regular structure of shock-accelerated $40-100 \mathrm{keV}$ electrons in the high latitude heliosphere, Astron. Astrophys., 316, 418-486, 1996.

Simnett G. M., and E. C. Roelof, Reverse shock acceleration of electrons and protons at mid-heliolatitudes from 5.3-3.8 AU, Space Sci. Rev., 72, 303-308, 1995.

Simnett G. M., K. Sayle, E. C. Roelof, Differences between the $0.35-1 \mathrm{MeV} / \mathrm{N} \mathrm{h} / \mathrm{He}$ ratio in solar and corotaing events at high heliolatitudes, Geophys. Res. Lett., 22, 3365-3368, 1995.

Van Hollebeke M. A. I., F. B. McDonald, J. H. Trainor, T. T. von Rosenvinge, The radial variation of corotating energetic particle streams in the inner and outer solar system, J. Geophys. Res., 83, 4723-4731, 1978. 